\title{
Nanomaterials for improved analytical processes
}

\author{
M. Valcárcel • B. M. Simonet
}

Published online: 22 October 2010

(C) Springer-Verlag 2010

One of the main goals of analytical science is to develop new methods in order to improve existing ones or meet the demands for (bio)chemical information posed by new social and economic problems. The growing demand for (bio) chemical information requires the development not only of tangible, but also of intangible tools to provide support for new analytical processes in routine (bio)analytical laboratories and to meet stringent scientific and technical quality requirements such as those of analytical laboratories involved in research, development and innovation activities. These two scenarios require a different approach in designing new or better analytical processes.

Improvements in analytical processes can be measured in terms of quality indicators named "analytical properties" that are used to characterize both the analytical process and its results. Analytical properties can be of three different types, namely (1) capital properties of the analytical results (accuracy and representativeness), (2) basic properties (precision, robustness, selectivity and sensitivity), which provide essential support to the capital properties and are characteristic of the process, and (3) productivity-related properties (expeditiousness, costs-effectiveness, safety), which pertain to the analytical process. These properties have complementary or mutually antagonistic relationships that should be considered in adopting optimal "quality compromises" between metrological and problem-solving objectives when designing and developing new (bio) analytical processes.

M. Valcárcel $(\bowtie) \cdot B$. M. Simonet

Department of Analytical Chemistry,

Marie Curie Annex Building, Campus de Rabanales,

University of Córdoba,

14071 Córdoba, Spain

e-mail: qa1meobj@uco.es
The use of nanotechnological tools in analytical methods helps to improve analytical properties including simplification as well as the possibility to carry out new types of analysis, for example bioimaging. This is the main reason why nanotechnological tools should be studied, developed and incorporated in analytical sciences.

Analytical nanoscience and nanotechnology currently provide one of the most promising avenues for developments in analytical science, derived from their two main fields of action, namely the analysis of nanostructured materials and their use as tangible tools.

Analysing the nanoworld in close cooperation with other scientists is, no doubt, a very ambitious goal for analytical chemistry. In fact, it is far from easy to extract reliable physicochemical information from nanostructured materials. Analytical and Bioanalytical Chemistry has published two special issues on this topic in the past: "Analytical tools for the nanoworld", with Renato Zenobi as guest editor (Vol. 396/1, January 2010), and "Chemical characterization of engineered nanoparticles", with Michael Winchester, Ralph Sturgeon and José Manuel Costa-Fernández as guest editors (Vol. 396/3, February 2010). One must emphasize the significance of the chemical approach to supplementing the information provided by typical physical techniques such as atomic force microscopy, electron microscopy and scanning tunnelling microscopy. Also, the growing use of spectrometric techniques such as Raman spectroscopy, near-field microscopy, laser ablation mass spectrometry, ion beams and nano-optical sensing at the nanoscale level in this context have conferred some analytical techniques with nanoscale resolution.

The use of nanomaterials as analytical tools is another major goal of analytical nanoscience and nanotechnology, and is the subject matter of this special issue. In practical terms, nanotechnology-based analytical processes can exploit the 
nanosize and exceptional properties of structured nanomatter. Nanometric analytical systems, which are based on nanosize and/or nanofluidics, are best exemplified by nano-chip liquid chromatography systems using flow rates as low as a few nanolitres per minute - some authors, however, have placed them outside the scope of nanotechnology. Nanotechnological analytical systems, which exploit the exceptional physicochemical properties of nanomaterials, account for most current uses of analytical nanoscience (particularly those involving nanoparticles for analytical purposes). Successful integration of the previous two types of systems into analytical nanosystems (e.g. molecular switches for the analytical process, individual carbon nanotubes for use as electrodes, or as replacements for conventional silica tips in atomic force microscopes) is still at an incipient stage.

The analytical process connecting a gross sample to its results essentially involves four steps, namely sample treatment, chromatographic and/or electrophoretic separation, detection, and data acquisition and processing. The role nanoparticles can play in the analytical process differs between steps. Nanomaterials are probably most useful for detection by virtue of their ability to replace conventional materials and also because of the advantages of electrochemical biosensors. Thus, nanoparticles can help stabilize biomolecules, increase active surfaces and facilitate electron transfer. Also, the exceptional optical properties of metallic nanoparticles derived from their plasmon resonance, and the photofluorescent properties of semiconducting nanoparticles (quantum dots), have fostered the development of optical nanosensors. Sample preparation has also profited from nanomaterials, albeit to a lesser extent than detection. For example, nanomaterials can advantageously replace conventional sorbents for solid-phase extraction and solid-phase microextraction. This is particularly so with carbon nanoparticles thanks to their especial $\pi$-electron configuration, and for metal oxides by virtue of their high surface area. In addition, nanostructured materials are being increasingly used as stationary phases in gas and liquid chromatography or electrochromatography, and also as pseudo-stationary phases in electrokinetic chromatography. This multiplicity of uses is well illustrated by the reviews and research papers in this special issue. Finally, we would like to stress the increasing importance of hybrid nanoparticles in the development of analytical tools, a field where their unique properties can open up very promising prospects for analytical nanoscience and nanotechnology.

We would like to thank Springer for giving us the opportunity to act as guest editors for this special issue and the authors for their contributions.

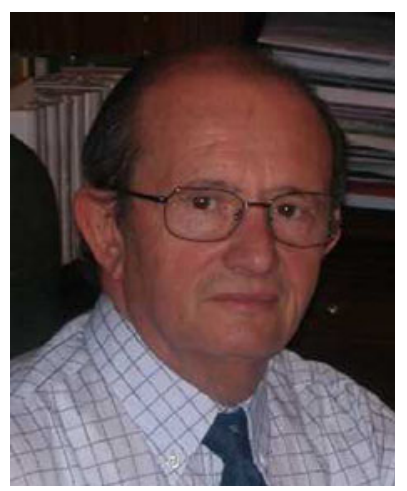

Miguel Valcárcel has been full Professor of Analytical Chemistry at the University of Córdoba (Spain) since 1976. He is the author and co-author of 800 scientific articles (in indexed journals), seven scientific monographs, eight textbooks and 16 book chapters. He has been the coordinator of 25 Spanish and 14 international research projects, as well as having 12 contracts with private firms and acting as a promoter of a spinoff devoted to nanotechnology. $\mathrm{He}$ has been a co-supervisor of 70 doctoral theses and an invited lecturer at more than 70 international meetings. He is the recipient of several scientific national (e.g. Award in Chemistry in Spain, 2005) and international (e.g. Robert Boyle Medal of the Royal Society of Chemistry, UK, 2004) prizes and is a member of the Spanish Royal Academy of Sciences.

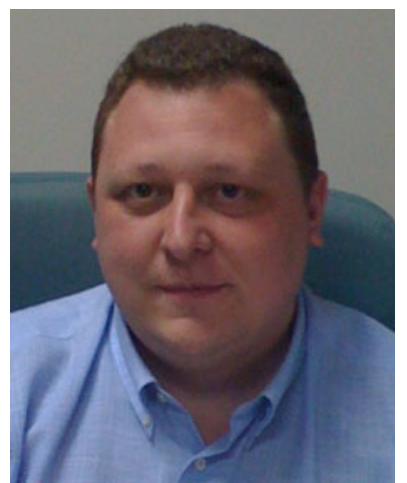

Bartolomé M. Simonet is a senior researcher at the University of Córdoba. He is the author and co-author of 80 scientific articles, two textbooks and five book chapters. He has been a co-supervisor of four doctoral theses and presently he is supervising seven doctoral theses. He has participated in five scientific research projects and is the scientific director of a spin-off devoted to nanotechnology. He has presented 21 oral communications at international meetings 